\title{
Cost-optimisation of screening for latent tuberculosis in close contacts
}

\section{To the Editors:}

We read with great interest the article by DiEL et al. [1] in a recent issue of the European Respiratory Journal. We believe that the authors have been successful in modelling and analysing the cost of alternatives for tuberculosis (TB) contact investigation. Nevertheless, we have some hesitation regarding a simplifying assumption that the authors have made in the follow-up of subjects after a positive screening test.

According to the contact investigation protocol modelled by DIEL et al. [1], close contacts with positive initial screening should undergo a chest radiograph, and those who do not receive treatment for latent infection may be followed up with two additional chest radiographs. A probability of $1 \%$ was assigned for suspicion of active TB in the follow-up of a positive screening test in all screening strategies. Several studies suggest that the sensitivity of QuantiFERON-TB Gold (QFT-G) in detecting latent or active TB is similar to (if not higher than) that of the tuberculin skin test (TST) [2-4], and its specificity is significantly higher, as QFT-G is not affected by previous bacille Calmette-Guérin (BCG) vaccination [2, 5]. This implies that QFT-G must have a remarkably higher positive predictive value in the presence of a high rate of previous BCG vaccination, and therefore that a higher number of subjects should undergo further work-up for the detection of TB after a positive QFT-G test. In the data provided by the authors, the number of positive TST results is 4.4 times as high as the number of positive QFT-G results (137 versus 31). Assuming that QFT-G and TST will result in the same rate of active case detection in the long run (an assumption upon which the cost minimisation analysis is justified), the positive predictive value of QFT-G for detection of active TB must be at least quadruple that of TST. The positive predictive value of the combined tests in other branches of the model should even be higher. This is in contrast with the authors' approach in assigning similar probabilities of suspecting TB in all branches of the decision tree.

Fortunately, since subjects suspected of having tuberculosis exit the model immediately, this is a conservative assumption in favour of the tuberculin skin test. Therefore, the overall conclusion of the study that QuantiFERON-TB Gold is less costly than the tuberculin skin test remains valid. Nevertheless, the calculated level of cost savings and also the rank order of the alternative strategies might be affected.

\section{Sadatsafavi and M. Najafzadeh}

Centre for Clinical Epidemiology and Evaluation, Vancouver General Hospital, Vancouver, Canada.

\section{REFERENCES}

1 Diel R, Nienhaus A, Lange C, Schaberg T. Cost-optimisation of screening for latent tuberculosis in close contacts. Eur Respir J 2006; 28: 35-44.
2 Mori T, Sakatani M, Yamagishi F, et al. Specific detection of tuberculosis infection: an interferon- $\gamma$-based assay using new antigens. Am J Respir Crit Care Med 2004; 170: 59-64.

3 Ferrara G, Losi M, Meacci M, et al. Routine hospital use of a new commercial whole blood interferon- $\gamma$ assay for the diagnosis of tuberculosis infection. Am J Respir Crit Care Med 2005; 172: 631-635.

4 Ferrara G, Losi M, D'Amico R, et al. Use in routine clinical practice of two commercial blood tests for diagnosis of infection with Mycobacterium tuberculosis: a prospective study. Lancet 2006; 367: 1328-1334.

5 Kang YA, Lee HW, Yoon HI, et al. Discrepancy between the tuberculin skin test and the whole-blood interferon $\gamma$ assay for the diagnosis of latent tuberculosis infection in an intermediate tuberculosis-burden country. JAMA 2005; 293: 2756-2761.

DOI: 10.1183/09031936.00094906

\section{From the authors:}

I would like to thank M. Sadatsafavi and M. Najafzadeh for raising an interesting concern. First, our cost minimisation analysis [1] was not a cost-effectiveness analysis between the consequences of isoniazid treatment and nontreatment over several decades; it only considered costs that accumulated over the 2 yrs following the last possible moment of exposure of the contact person to the infectious source case.

Secondly, we had to include new tuberculosis (TB) cases that were typically identified in large contact studies in our model. These generally equate to $\sim 1 \%$ of the contact persons investigated, and, because they do not result in further investigation costs, must be shown to drop out of the cohort under suspicion. In our model, we distributed this "dropping out" over the entire 2-yr period. The crucial point, however, is simple: previous molecular-epidemiological studies [2-4] have shown that two types of TB cases may be expected in contact investigations of the kind we studied. On the one hand, there are TB cases with no epidemiological connection to the source case in question, i.e. they must have been infected by another source case at the same time or even many years prior to their diagnosis. On the other hand, there are secondary cases (reactivations) of infections that are actually acquired from the source case. Only these cases could be directly assigned to contacts that had previously tested positive using the tuberculin skin test or QuantiFERON-gold. Depending on the risk profile of the source case and its contacts, the proportions of the two types will vary and cannot be predicted.

Thus, although we found a number of positive tuberculin skin tests 4.4 times higher than positive QuantiFERON-gold results, we currently do not know whether the positive predictive value of the QuantiFERON-gold is really higher 
than that of a tuberculin skin test not confounded by Bacille Calmette-Guérin vaccination or nontuberculous mycobacteria. This must be determined by ongoing prospective restriction fragment length polymorphism studies observing the clinical course of QuantiFERON-gold-positive contact persons. These use "clustering" with the source case and observe who becomes infected with the disease. As QuantiFERON-gold is only just being implemented for routine use in western industrial countries, this question is not sufficiently answered. In our view, it would be speculative to distinguish between the predictive values of the two testing procedures. Therefore, we assigned a uniform probability of re-activation to manifest tuberculosis disease to subjects testing positive to either the tuberculin skin test or the QuantiFERON-gold assay.

\section{R. Diel}

Heinrich-Heine-University, Postfach 101007, Düsseldorf 40001, Germany.

\section{REFERENCES}

1 Diel R, Nienhaw A, Lange C, Schaberg T. Cost-optimisation of screening for latent tuberculosis in close contacts. Eur Respir J 2006; 28: 35-44.

2 Behr A, Hopewell PC, Paz EA, Kamamura LM, Schecter GF, Small PM. Predictive value of contact investigation for identifying recent transmission of Mycobacterium tuberculosis. Am J Respir Crit Care Med 1998; 158: 465-469.

3 Diel R, Schneider S, Meywald-Walter K, Ruf CM, RüschGerdes S, Niemann S. Epidemiology of tuberculosis in Hamburg, Germany: long-term population-based analysis applying classical and molecular epidemiological techniques. J Clin Microbiol 2002; 40: 532-539.

4 Diel R, Meywald-Walter K, Gottschalk R, Rüsch-Gerdes S, Niemann S. Ongoing outbreak of tuberculosis in a lowincidence community: a molecular-epidemiological evaluation. Int J Tuberc Lung Dis 2004; 8: 855-861.

\section{Therapy of exophytic bronchial tumorous stenosis by flexible cryoprobe}

\section{To the Editors:}

We read with interest the article by VERGNON et al. [1] in the European Respiratory Journal, about the place of cryotherapy, brachytherapy and photodynamic therapy in the therapeutic bronchoscopy of lung cancers. The article describes indications and limitations of cryotherapy. In the opinion of VERGNON et al. [1], the effect of cryotherapy is delayed and is, therefore, not useful for the immediate debulking of an obstructive endoluminal bronchial tumour. For these cases, VERGNON et al. [1] advise coring of the tumour with the rigid bronchoscope and subsequent laser therapy or electrocautery. The indication for cryotherapy in this setting is seen as a treatment option for any remaining infiltrative parts of the tumour into the bronchial wall, but not as a treatment option of first choice to remove the main part of an exophytic tumour.

In our clinic, we have positive experience in a large number of patients with the application of cryotherapy for the immediate treatment of acute dyspnoea in patients with obstructive malignant bronchial tumours. We use the cryorecanalisation technique with the rigid bronchoscope under general anaesthesia, as well as with a flexible technique during local anaesthesia and sedation. The commercially available flexible cryoprobe (ERBE, Tübingen, Germany) we use is $780 \mathrm{~mm}$ in length and $2.3 \mathrm{~mm}$ in diameter. The probe's tip is cooled to $-89.5^{\circ} \mathrm{C}$ by sudden gas decompression (nitrous oxide) in the probe's head. The cryoprobe is a closed system, which can be re-sterilised and re-used. In many cases, we use the cryorecanalisation method in combination with argon plasma coagulation for a more immediate treatment of diffuse tumour bleeding after coring with the rigid bronchoscope.
We apply the following technique: 1) the flexible cryoprobe is introduced via a flexible bronchoscope in combination with an Ikeda tubus or a rigid bronchoscope; 2) the tip of the cryoprobe is pushed into the protruding exophytic tumour mass and freezing is started for $\leqslant 5 \mathrm{~s}$ with a footpad; 3 ) the cryoprobe, including the flexible bronchoscope, is abruptly mechanically removed, a tumour mass of 3-5 $\mathrm{mm}$ is frozen at the tip of the cryoprobe and can easily be extracted; 4) rapid thawing is induced in a water bath for $\leqslant 5 \mathrm{~s}$; and 5) the procedure is repeated until the tumour mass has been extracted and the target bronchus is re-opened. If necessary, subsequent stent implantation is performed in the same session without any problems. With this technique, we have seen no severe bleedings if coagulation parameters were within the normal range. Overall, we have a very low complication rate. Severe bleeding during re-canalisation with the cryoprobe is very rare, we have never seen perforations, and the primary success rate of re-canalisation is very high $(>90 \%)$, even in longdistance stenoses, and in our experience comparable to that of laser beam or electrocautery. The feasibility of this method for the immediate management of acute airway obstruction has already been published [2]. In contrast to the cryotherapy technique described by VERGNON et al. [1], which requires a second bronchoscopy after 8-10 days for the removal of necrotic tissue, a second-look bronchoscopy is not mandatory with our technique.

In addition, the specimens obtained by cryorecanalisation for diagnostic purposes are much larger and of excellent tissue quality, without squeezing artefacts, compared with specimens extracted using forceps. In a study with 60 patients, comparing 